\title{
String model calculation of the strongly intensive observables for multiplicities in two windows
}

\author{
Vladimir Vechernin ${ }^{1, *}$ \\ ${ }^{1}$ Saint Petersburg State University, Saint Petersburg, Russia
}

\begin{abstract}
We calculate the strongly intensive observables for multiplicities in two rapidity windows in the model with independent identical strings taking into account the charge sign of particles. We express the observables through the string pair correlation functions describing the correlations between the same and opposite sign particles produced in a string decay. We extract these charge-wise string two-particle correlation functions from the ALICE data on the forward-backward correlations and the balance function. Using them we predict the behavior of the charge-wise strongly intensive observables in the model with independent identical strings.
\end{abstract}

\section{Introduction}

It is known that the investigations of long range rapidity correlations give the information about the initial stage of high energy hadronic interactions [1]. So, to find a signature of the string fusion and percolation phenomenon [2-4] in ultrarelativistic heavy-ion collisions, the study of the correlations between multiplicities in two separated rapidity intervals, known as the forward-backward (FB) multiplicity correlations, was proposed [5].

Later it was realized [6-8] that the investigations of the FB correlations involving intensive observables in forward and backward observation windows as, e.g., the event-mean transverse momentum, enable to suppress the contribution of trivial "volume" fluctuations originating from fluctuations in the number of initial sources (strings) and to obtain a more clear signal on the process of string fusion, compared to usual FB multiplicity correlations.

In the present work, we explore another way to suppress the contribution of the "volume" fluctuations, passing to the more sophisticated correlation observable. In quark-gluon string model we calculate the strongly intensive variable

$$
\Sigma\left(n_{F}, n_{B}\right) \equiv\left[\left\langle n_{F}\right\rangle \omega_{n_{B}}+\left\langle n_{B}\right\rangle \omega_{n_{F}}-2 \operatorname{cov}\left(n_{F} n_{B}\right)\right] /\left[\left\langle n_{F}\right\rangle+\left\langle n_{B}\right\rangle\right]
$$

for the charged particles multiplicities $n_{F}$ and $n_{B}$ in forward and backward rapidity windows, introduced like in $[9,10]$ to suppress the contribution of the "volume" fluctuations in hadronic interactions at high energies $\left(\omega_{n}=D_{n} /\langle n\rangle\right.$ is a scaled variance).

We express this observable through the fundamental characteristics of a string: the multiplicity $\mu_{0}$ per unit of rapidity and the two-particle correlation function $\Lambda\left(\eta_{1}-\eta_{2}\right)$, describing the fragmentation of a single string (see formula (6) below). We confirm the strongly intensive character of the observable in the case with fluctuating number of identical strings. It

\footnotetext{
*e-mail: v.vechernin@spbu.ru
} 
does not depend on the average number of strings, nor on the magnitude of event-by-event fluctuation of their number.

We also consider the strongly intensive observable (1) taking into account the particle charge sign. In the case of charge symmetry, which is a very good approximation for the mid-rapidity region at LHC collision energies, these charge-wise strongly intensive observables are expressed through the string pair correlation functions for particles of the same and opposite signs, $\Lambda^{\text {same }}(\Delta \eta)$ and $\Lambda^{o p p}(\Delta \eta)$. We obtain the information about these two functions from the ALICE data on the FB correlations [11, 12] and the balance function [13], using the relations (11) and (19). Finally, using these two-particle correlation functions of a string, we calculate the charge-wise strongly intensive observables $\Sigma\left(n_{F}^{+}, n_{B}^{+}\right), \Sigma\left(n_{F}^{-}, n_{B}^{+}\right)$and $\Sigma\left(n_{F}^{+}, n_{F}^{-}\right)$.

\section{The model with independent identical strings}

In this paper, we restrict our consideration to a simple case of the model with independent identical strings [14]. In this model, we suppose that the number of strings $N$ fluctuates event by event around some mean value $\langle N\rangle$ with some scaled variance $\omega_{N}=D_{N} /\langle N\rangle$.

To characterize the properties of a single string, we introduce the single and double distributions of particles produced from a single string fragmentation and the string two-particle correlation function defined in the standard way (see e.g. [15]):

$$
\lambda(\eta) \equiv \frac{d N_{c h}}{d \eta}, \quad \lambda_{2}\left(\eta_{1}, \eta_{2}\right) \equiv \frac{d^{2} N_{c h}}{d \eta_{1} d \eta_{2}}, \quad \Lambda\left(\eta_{1}, \eta_{2}\right) \equiv \frac{\lambda_{2}\left(\eta_{1}, \eta_{2}\right)}{\lambda\left(\eta_{1}\right) \lambda\left(\eta_{2}\right)}-1 .
$$

In mid-rapidity region at LHC energies, we assume the translation invariance in rapidity for the string characteristics. Then

$$
\lambda(\eta)=\mu_{0}, \quad \lambda_{2}\left(\eta_{1}, \eta_{2}\right)=\lambda_{2}\left(\eta_{1}-\eta_{2}\right), \quad \Lambda\left(\eta_{1}, \eta_{2}\right)=\Lambda\left(\eta_{1}-\eta_{2}\right) .
$$

For symmetric $2 \pi$-azimuth rapidity observation windows $\delta \eta_{F}=\delta \eta_{B} \equiv \delta \eta$ and a symmetric reaction, the definition of the strongly intensive variable (1) can be simplified to

$$
\Sigma\left(n_{F}, n_{B}\right)=\omega_{n}-\operatorname{cov}\left(n_{F}, n_{B}\right) /\langle n\rangle=\left[D_{n}-\operatorname{cov}\left(n_{F}, n_{B}\right)\right] /\langle n\rangle=\left[\left\langle n^{2}\right\rangle-\left\langle n_{F} n_{B}\right\rangle\right] /\langle n\rangle,
$$

where we imply that the observation windows $\delta \eta_{F}$ and $\delta \eta_{B}$ are separated by a rapidity gap $\eta_{\text {gap }}$, which corresponds to the distance $\Delta \eta=\eta_{\text {gap }}+\delta \eta$ between their centers. It is clear that for the symmetric reaction we have $\left\langle n_{F}\right\rangle=\left\langle n_{B}\right\rangle \equiv\langle n\rangle$ and $\omega_{n_{F}}=\omega_{n_{B}} \equiv \omega_{n}$.

For small observation windows of width $\delta \eta \ll \eta_{\text {corr }}$ where the $\eta_{\text {corr }}$ is the characteristic correlation length for particles produced from the same string, we have shown in [12] that in the framework of the model with independent identical strings:

$$
\omega_{n}=D_{n} /\langle n\rangle=1+\mu_{0} \delta \eta\left[\Lambda(0)+\omega_{N}\right], \quad \operatorname{cov}\left(n_{F}, n_{B}\right) /\langle n\rangle=\mu_{0} \delta \eta\left[\Lambda(\Delta \eta)+\omega_{N}\right],
$$

where $\Delta \eta=\eta_{F}-\eta_{B}$ is a distance between the centers of the forward and backward observation windows. Then using Eq. (4), we find

$$
\Sigma\left(n_{F}, n_{B}\right)=1+\mu_{0} \delta \eta[\Lambda(0)-\Lambda(\Delta \eta)] .
$$

Eq. (6) means that in the framework of this model the observable $\Sigma\left(n_{F}, n_{B}\right)$ is strongly intensive. It is independent of both the mean number of strings $\langle N\rangle$ and its fluctuation $\omega_{N}$. It depends only on the string parameters $\mu_{0}, \Lambda(\Delta \eta)$ and the widths of observation windows $\delta \eta$. Whereas the scaled variance $\omega_{n}$ (Eq. 5) is intensive but not a strongly intensive observable, 
because although it is independent on the mean number of string $\langle N\rangle$, nevertheless through $\omega_{N}$ it depends on fluctuation of their number.

From the Eq. (6) we see also the main properties of the $\Sigma\left(n_{F}, n_{B}\right)$ expected in this model. Starting from the value 1 it increases with a distance $\Delta \eta$ between the centers of the observation windows, since the two-particle correlation function of a string $\Lambda(\Delta \eta)$ decreases with $\Delta \eta$. The extent of $\Sigma(\Delta \eta)$ increase with $\Delta \eta$ is proportional to the width of the observation windows $\delta \eta$. More detailed description of the $\Sigma\left(n_{F}, n_{B}\right)$ needs the knowledge of the two-particle correlation function of a string $\Lambda(\Delta \eta)$.

\section{The $\Lambda(\Delta \eta)$ from forward-backward correlations}

In our paper [12], this function was fit using the experimental pp ALICE data [11] on FB correlations between multiplicities in windows separated in rapidity and azimuth at three initial energies in the framework of the model with independent identical strings:

$$
\Lambda(\Delta \eta, \Delta \phi)=\Lambda_{1} e^{-\frac{|\Delta \eta|}{\eta_{1}}} e^{-\frac{\Delta \phi^{2}}{\varphi_{1}^{2}}}++\Lambda_{2}\left(e^{-\frac{\left|\Delta \eta-\eta_{0}\right|}{\eta_{2}}}+e^{-\frac{\left|\Delta \eta+\eta_{0}\right|}{\eta_{2}}}\right) e^{-\frac{(\Delta \phi \phi \mid-\pi)^{2}}{\varphi_{2}^{2}}}
$$

together with the value of scaled variance of the number of strings $\omega_{N}$. The values of the parameters are shown in Tab. 1 in the paper [12]. It should be recalled that the comparison of the model with experimental data in [12] enables to fix only the product of the parameters $\mu_{0} \Lambda_{1}, \mu_{0} \Lambda_{2}$ and $\mu_{0} \omega_{N}$. In (7) we imply that $|\Delta \phi| \leq \pi$. The periodic extension is implied for $|\Delta \phi|>\pi$.

Our string two-particle correlation functions (2) defined for $2 \pi$-azimuth observation windows can be obtained by simple averaging over azimuth (see [12]):

$$
\Lambda(\Delta \eta)=\frac{1}{\pi} \int_{0}^{\pi} \Lambda(\Delta \eta, \Delta \phi) d \Delta \phi
$$

So, integrating the fit (7) we find $\mu_{0} \Lambda(\Delta \eta)$ shown below by triangulares in Fig. 1.

\section{$4 \Sigma$ with charges}

In Sec. 1, we introduce the strongly intensive observable $\Sigma$ based on multiplicities of the all charged hadrons measured in two rapidity intervals. Here we consider various combinations of electric charges in these windows and similarly to Eq. (1) we can determine $\Sigma\left(n_{F}^{+}, n_{B}^{+}\right)$, $\Sigma\left(n_{F}^{-}, n_{B}^{-}\right), \Sigma\left(n_{F}^{+}, n_{B}^{-}\right)$and $\Sigma\left(n_{F}^{-}, n_{B}^{+}\right)$. We can also introduce an additional strongly intensive observable that measures the correlation between multiplicities of different charges in the same window $\Sigma\left(n_{F}^{+}, n_{F}^{-}\right)$and $\Sigma\left(n_{B}^{+}, n_{B}^{-}\right)[16]$.

For symmetric reaction and symmetric windows $\left(F \rightleftarrows B\right.$ invariance), we have $\left\langle n_{F}^{+}\right\rangle=$ $\left\langle n_{B}^{+}\right\rangle \equiv\left\langle n^{+}\right\rangle, \omega_{n_{F}^{+}}=\omega_{n_{B}^{+}} \equiv \omega_{n^{+}}$and the same for $n^{-}$. In this case, we have also $\operatorname{cov}\left(n_{F}^{+}, n_{F}^{-}\right)=$ $\operatorname{cov}\left(n_{B}^{+}, n_{B}^{-}\right), \operatorname{cov}\left(n_{F}^{+}, n_{B}^{-}\right)=\operatorname{cov}\left(n_{F}^{-}, n_{B}^{+}\right)$.

In case of additional charge symmetry ( $+\rightleftarrows-$ invariance), we have $\left\langle n^{+}\right\rangle=\left\langle n^{-}\right\rangle=\langle n\rangle / 2$, $\omega_{n^{+}}=\omega_{n^{-}}, \operatorname{cov}\left(n_{F}^{+}, n_{B}^{+}\right)=\operatorname{cov}\left(n_{F}^{-}, n_{B}^{-}\right)$, which is a very good approximation for the midrapidity region at LHC collision energies. In this approximation, we have for the distributions and two-particle correlation functions describing decay properties of a string:

$$
\begin{gathered}
\lambda^{+}(\eta)=\lambda^{-}(\eta)=\lambda(\eta) / 2=\mu_{0} / 2, \\
\Lambda^{++}(\Delta \eta)=\Lambda^{--}(\Delta \eta) \equiv \Lambda^{\text {same }}(\Delta \eta), \quad \Lambda^{+-}(\Delta \eta)=\Lambda^{-+}(\Delta \eta) \equiv \Lambda^{o p p}(\Delta \eta)
\end{gathered}
$$


where we also take into account the translation invariance, which takes place in the midrapidity region at LHC energies. It is easy to check that by definition (2)

$$
\Lambda(\Delta \eta)=\left[\Lambda^{o p p}(\Delta \eta)+\Lambda^{\text {same }}(\Delta \eta)\right] / 2 .
$$

For small observation windows, we find in this approximation that:

$$
\begin{gathered}
\Sigma\left(n_{F}^{+}, n_{B}^{+}\right)=1+\mu_{0} \delta \eta\left[\Lambda^{\text {same }}(0)-\Lambda^{\text {same }}(\Delta \eta)\right] / 2, \\
\Sigma\left(n_{F}^{+}, n_{B}^{-}\right)=1+\mu_{0} \delta \eta\left[\Lambda^{\text {same }}(0)-\Lambda^{o p p}(\Delta \eta)\right] / 2, \\
\Sigma\left(n_{F}^{+}, n_{F}^{-}\right)=1+\mu_{0} \delta \eta\left[\Lambda^{\text {same }}(0)-\Lambda^{o p p}(0)\right] / 2 .
\end{gathered}
$$

We see that as expected by analogy with Eq. (6) the $\Sigma\left(n_{F}^{+}, n_{B}^{+}\right) \rightarrow 1$ at $\Delta \eta \rightarrow 0$, however the $\Sigma\left(n_{F}^{+}, n_{B}^{-}\right)$tends to be equal to $\Sigma\left(n_{F}^{+}, n_{F}^{-}\right)$and not to 1 in this limit. Note that combining (12-14) and using Eqs. (6) and (11), we can obtain the following relation

$$
\Sigma\left(n_{F}, n_{B}\right)=\Sigma\left(n_{F}^{+}, n_{B}^{+}\right)+\Sigma\left(n_{F}^{+}, n_{B}^{-}\right)-\Sigma\left(n_{F}^{+}, n_{F}^{-}\right)
$$

\section{Connection with balance function}

To obtain the correlation functions $\Lambda^{\text {same }}(\Delta \eta)$ and $\Lambda^{o p p}(\Delta \eta)$ separately, we need some additional experimental information since the FB correlations used above depend according to Eq. (11) on the sum of these two correlation functions only. We use for this purpose the recent results obtained by the ALICE collaboration for the so-called balance function $B(\Delta \eta, \Delta \phi)$ [13]. In this paper, the balance function is supposed to be proportional to the difference between unlike-sign and like-sign two-particle correlation functions:

$$
B(\Delta \eta, \Delta \phi) \equiv\left[C_{+-}+C_{-+}-C_{++}-C_{--}\right] / 2,
$$

or under taken assumptions

$$
B(\Delta \eta, \Delta \phi)=C_{\text {opp }}-C_{\text {same }} .
$$

The rapidity projection $B(\Delta \eta)$ is defined in this paper as

$$
B(\Delta \eta) \equiv \int_{-\frac{\pi}{2}}^{\frac{\pi}{2}} B(\Delta \eta, \Delta \phi) d \Delta \phi+\int_{\frac{\pi}{2}}^{\frac{3 \pi}{2}} B(\Delta \eta, \Delta \phi) d \Delta \phi .
$$

Taking into account the normalization of the two-particle correlation functions used in paper [13], we find

$$
B(\Delta \eta)=\mu_{0}\left[\Lambda^{o p p}(\Delta \eta)-\Lambda^{\text {same }}(\Delta \eta)\right] / 4
$$

Note that the authors of this paper, defining by the so-called di-hadron correlation method the two-particle correlation functions and entering the definition of balance function (16), impose the requirement that the transverse momentum of the "trigger" particle must be higher than the "associated" one. If this requirement is not imposed, then the balance function values will be two times higher (see the remark before the Subsec. 6.1.1 in the paper [13]), what corresponds to the coefficient $1 / 2$ in (19) instead of $1 / 4$. (On correspondence of different definitions of two-particle correlation functions, see e.g. [12]).

Using Eqs. (11) and (19), we can find the correlation functions $\Lambda^{\text {same }}(\Delta \eta)$ and $\Lambda^{o p p}(\Delta \eta)$ separately performing the simultaneous fit of the experimental data obtained in pp collisions at $7 \mathrm{TeV}$ for the balance functions $B(\Delta \eta)$ [13] and of $\mu_{0} \Lambda(\Delta \eta)$ extracted from the FB correlations [11] (see Sec. 3). Since the FB correlations were measured experimentally for minimum 


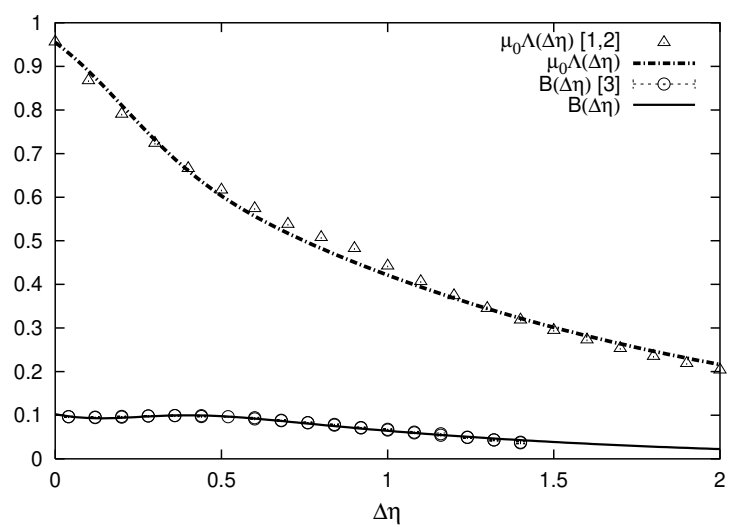

Figure 1. The results of simultaneous fitting (lines) by formulas (11) and (19) of the string twoparticle correlation function $\mu_{0} \Lambda(\Delta \eta)$ (triangles), extracted in [12] from the ALICE data [11] on the FB correlations (see Section 3), and the balance function $B(\Delta \eta)$ (circles) measured by ALICE [13] in pp collisions at $7 \mathrm{TeV}$

Table 1. The value of the parameters in formulas (20) and (21) for the charge-wise correlation functions of a string $\Lambda^{o p p}(\Delta \eta)$ and $\Lambda^{\text {same }}(\Delta \eta)$ obtained by the fit procedure, which is presented in Fig. 1

\begin{tabular}{|c|c|c|c|}
\hline$a$ & opp & same & $H B T$ \\
\hline \hline$\mu_{0} \Lambda_{0}^{a}$ & 1.16 & 0.5 & 0.25 \\
$\eta_{a}$ & 1.34 & 1.87 & 0.33 \\
\hline
\end{tabular}

bias pp events, the balance functions for the $70-80 \%$ pp centrality class were used assuming that the minimum bias is dominated by peripheral collisions.

We use the simplest fit for the unlike-sign two-particle correlation function of a string:

$$
\Lambda^{o p p}(\Delta \eta)=\Lambda_{0}^{o p p} \exp \left(-|\Delta \eta| / \eta_{o p p}\right)
$$

To better fit the like-sign two-particle correlation function, we have to take into account the HBT correlation term, which is important at small values of $\Delta \eta$ :

$$
\Lambda^{\text {same }}(\Delta \eta)=\Lambda_{0}^{\text {same }} \exp \left(-|\Delta \eta| / \eta_{\text {same }}\right)+\Lambda_{0}^{H B T} \exp \left[-\left(\Delta \eta / \eta_{H B T}\right)^{2}\right] .
$$

The results of the simultaneous fit of the string two-particle correlation function $\mu_{0} \Lambda(\Delta \eta)$, (11) extracted in [12] from the ALICE data [11] on FB correlations and the balance function $B(\Delta \eta)(19)$ measured by ALICE [13] in pp collisions at $7 \mathrm{TeV}$ are presented in Fig. 1. This fit fixes the parameters in formulas (20) and (21) for the charge-wise correlation functions of a string: $\Lambda^{o p p}(\Delta \eta)$ and $\Lambda^{\text {same }}(\Delta \eta)$, as presented in Tab. 1. We see that the correlation length between opposite charge particles $\eta_{\text {opp }}$ is smaller then the one between same charge particles $\eta_{\text {same }}$ as expected due to local charge conservation in a string fragmentation process.

\section{Results}

Using the found charge-wise correlation functions of a string $\Lambda^{o p p}(\Delta \eta)$ and $\Lambda^{\text {same }}(\Delta \eta)($ Eqs. (12), (14)), we can predict the behavior of the charge-wise strongly intensive observables $\Sigma\left(n_{F}^{+}, n_{B}^{+}\right), \Sigma\left(n_{F}^{+}, n_{B}^{-}\right)$and $\Sigma\left(n_{F}^{+}, n_{F}^{-}\right)$in the model with independent identical strings. In Fig. 2, these dependencies are presented for the case of two small observation windows as a function 


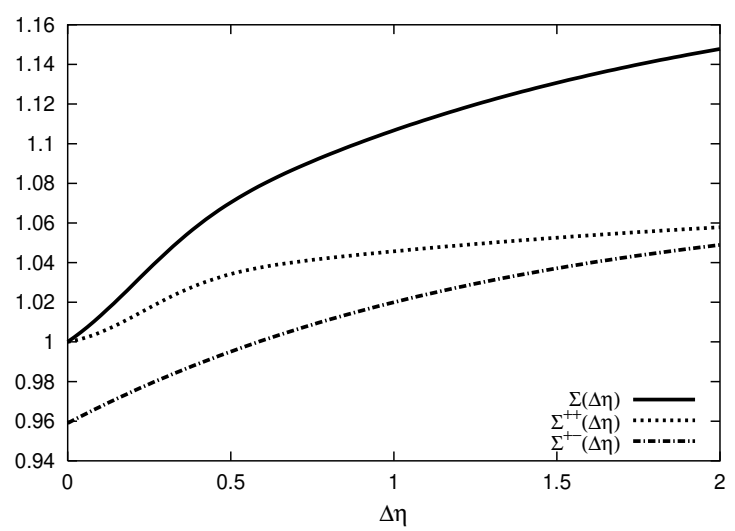

Figure 2. The charge-wise strongly intensive variables $\Sigma\left(n_{F}^{+}, n_{B}^{+}\right) \equiv \Sigma^{++}(\Delta \eta)$ (dotted line) and $\Sigma\left(n_{F}^{+}, n_{B}^{-}\right) \equiv \Sigma^{+-}(\Delta \eta)$ (dash-dotted line) for the case of two small observation windows as a function of rapidity gap $\Delta \eta$ between them. These variables were calculated using Eqs. (12) and (14) with the string correlation functions $\Lambda^{o p p}(\Delta \eta)$ and $\Lambda^{\text {same }}(\Delta \eta)$ obtained with the fit procedure shown in Fig. 1 . Full line shows the strongly intensive observable $\Sigma\left(n_{F}, n_{B}\right) \equiv \Sigma(\Delta \eta)$ for the full multiplicities in these windows given by Eq. (15)

of rapidity gap between them $\Delta \eta$. For comparison, the strongly intensive variable $\Sigma\left(n_{F}, n_{B}\right)$ for the full multiplicities in these windows given by the formula (15) is also presented in this figure.

We see that, as was noted in the end of Sec. $4, \Sigma\left(n_{F}^{+}, n_{B}^{-}\right) \rightarrow \Sigma\left(n_{F}^{+}, n_{F}^{-}\right)=0.96 \neq 1$ at $\Delta \eta \rightarrow 0$ whereas $\Sigma\left(n_{F}^{+}, n_{B}^{+}\right) \rightarrow 1$ similar to the total $\Sigma\left(n_{F}, n_{B}\right) \rightarrow 1$ for full multiplicities.

We see also that expressing the charge-wise strongly intensive observables for multiplicities in two rapidity windows through the string pair correlation functions describing the correlations between the same and opposite sign particles and extracting the last from the ALICE data on the FB correlations and the balance function, we can predict the behavior of the charge-wise strongly intensive observables in the framework of the model with independent identical strings.

Acknowledgement. The reported study was funded by RFBR according to the research project 18-02-40122 and the Saint-Petersburg State University outgoing academic mobility grant.

\section{References}

[1] A. Dumitru, F. Gelis, L. McLerran, R. Venugopalan, Nucl. Phys. A 810, 91 (2008)

[2] T.S. Biro, H.B. Nielsen, J. Knoll, Nucl. Phys. B 245, 449 (1984)

[3] A. Bialas, W. Czyz, Nucl. Phys. B 267, 242 (1986)

[4] M.A. Braun, C. Pajares, Phys. Lett. B 287, 154 (1992)

[5] N.S. Amelin et al., Phys. Rev. Lett. 73, 2813 (1994)

[6] ALICE collaboration et al., J. Phys. G 32(10), 1295 (2006)

[7] V.V. Vechernin, R.S. Kolevatov, Phys. Atom. Nucl. 70, 1809 (2007)

[8] V. Kovalenko, V. Vechernin, J. Phys.: Conf. Ser. 798, 012053 (2017)

[9] M.I. Gorenstein, M. Gazdzicki, Phys. Rev. C, 84, 014904 (2011)

[10] E.V. Andronov, Theor. Math. Phys., 185, 1383 (2015)

[11] J. Adam et al. (ALICE Collab.), JHEP 05, 097 (2015)

[12] V. Vechernin, Nucl. Phys. A 939, 21 (2015) 
[13] J. Adam et al. (ALICE Collab.), Eur. Phys. J. C. 76, 86 (2016)

[14] M.A. Braun, C. Pajares, V.V. Vechernin, Phys. Lett. B 493, 54 (2000)

[15] C. Pruneau, S. Gavin, and S. Voloshin, Phys. Rev. C 66, 044904 (2002)

[16] E. Andronov (for the NA61/SHINE Collab.), J. Phys.: Conf. Ser. 668, 012036 (2016) 